\title{
On the Orbital Properties of Millisecond Pulsar Binaries
}

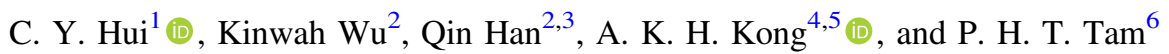 \\ ${ }^{1}$ Department of Astronomy and Space Science, Chungnam National University, Daejeon 34134, Republic of Korea; huichungyue@gmail.com, cyhui@cnu.ac.kr \\ ${ }^{2}$ Mullard Space Science Laboratory, University College London, Holmbury St. Mary, Surrey, RH5 6NT, UK \\ ${ }^{3}$ School of Astronomy and Space Science, Nanjing University, 163 Xianlin Avenue, Nanjing, Jiangsu 210093, People's Republic of China \\ ${ }^{4}$ Institute of Astronomy, National Tsing Hua University, Hsinchu, Taiwan \\ ${ }^{5}$ Astrophysics, Department of Physics, University of Oxford, Keble Road, Oxford OX1 3RH, UK \\ ${ }^{6}$ Institute of Astronomy and Space Science, Sun Yat-Sen University, Guangzhou 510275, People's Republic of China \\ Received 2017 November 1; revised 2018 July 18; accepted 2018 July 23; published 2018 August 29
}

\begin{abstract}
We report a detailed analysis of the orbital properties of a binary millisecond pulsar (MSP) with a white dwarf (WD) companion. Positive correlations between the orbital period $P_{\mathrm{b}}$ and eccentricity $\epsilon$ are found in two classes of MSP binaries with a He WD and with a CO/ONeMg WD, though their trends are different. The distribution of $P_{\mathrm{b}}$ is not uniform. Deficiency of sources at $P_{\mathrm{b}} \sim 35-50$ days (Gap 1) have been mentioned in previous studies. On the other hand, another gap at $P_{\mathrm{b}} \sim 2.5-4.5$ days (Gap 2) is identified for the first time. Inspection of the relation between $P_{\mathrm{b}}$ and the companion masses $M_{\mathrm{c}}$ revealed the subpopulations of MSP binaries with a He WD separated by Gap 1, above which $P_{\mathrm{b}}$ is independent of $M_{\mathrm{c}}$ (horizontal branch) but below which $P_{\mathrm{b}}$ correlates strongly with $M_{\mathrm{c}}$ (lower branch). A distinctive horizontal branch and a lower branch separated by Gap 2 were identified for the MSP binaries with a CO/ONeMg WD at shorter $P_{\mathrm{b}}$ and higher $M_{\mathrm{c}}$. Generally, $M_{\mathrm{c}}$ are higher in the horizontal branch than in the lower branch for the MSP binaries with a He WD. These properties can be explained in terms of a binary orbital evolution scenario in which the WD companion was ablated by a pulsar wind in the post-masstransfer phase.
\end{abstract}

Key words: binaries: general - pulsars: general

\section{Introduction}

Shortly after the first millisecond pulsar (MSP) PSR B1937 +21 (Backer et al. 1982) was discovered, a "recycling" scenario for its formation, in which old neutron stars in binaries were spun up by acquiring angular momentum through accreting material from a companion (Alpar et al. 1982; Radhakrishnan \& Srinivasan 1982; Fabian et al. 1983), was proposed. The process would occur at the late evolutionary stage of low-mass X-ray binaries (LMXBs). The scenario explains the low surface magnetic fields, the millisecond equilibrium rotational periods and the very low spinning-down rates as observed in MSPs (Backer et al. 1983). It received further support through the discovery of accreting millisecond X-ray pulsars (e.g., SAX J1808.4-3658, Wijnands \& van der Klis 1998) and "red-back" MSPs, which show alternating LMXB and rotation-powered states (e.g., PSR J1023+038, Thorstensen \& Armstrong 2005; Archibald et al. 2009, 2010; Takata et al. 2014). In spite of its success, certain evolutionary aspects of MSPs, especially those in binaries, have yet to be satisfactorily explained within the scenario's framework. Besides the initial distributions of the orbital separations and the companion masses, how the progenitor systems evolved through a common-envelope phase, which has not been directly observed, and how orbital angular-momentum was transported at various evolutionary stages are still unclear (Tauris 1996; Taam et al. 2000). The complex evolution dynamics of MSP binaries is also reflected by a "period gap" at $P_{\mathrm{b}} \sim 23-56$ days where there is a deficiency in source number (Tauris 1996; Taam et al. 2000). Its presence indicates a possible bifurcation process in operation, causing divergent evolutionary paths for the subpopulations of the systems (Tauris 1996).

Theoretical investigations have predicted the relations between the orbital properties of MSPs. Two relations of the
MSPs in a wide orbit of orbital period $P_{b} \gtrsim 2$ days with companion mass $M_{\mathrm{c}} \lesssim 2 M_{\odot}$ have long been suggested as the dynamical fossils of the spin-up era (Phinney \& Kulkarni 1994). These are $P_{b}-M_{\mathrm{c}}$ relation (Refsdal \& Weigert 1971; Tauris \& Savonije 1999) and that between $P_{b}$ and the eccentricity $\epsilon$ (Phinney 1992). Hence, a thorough population analysis of the MSP binaries can help us to gain new insights into the intricate evolutionary paths of MSP binaries. With the expanded sample established by the recent observations, we conducted a statistical analysis of orbital properties of MSP binaries with a white-dwarf (WD) companion. This article reports our findings and interpretations in light of the new statistics.

\section{Data Analysis}

We focus on the MSP binaries with a WD companion, adopting the following selection criteria: (i) the MSP rotational periods $P<40 \mathrm{~ms}$ and (ii) the sources are in the Galactic field. MSP binaries in globular clusters (GC), which had involved different dynamical formation processes (see Hui et al. 2010), were excluded. The system parameters of the sources were derived from the data in the updated ATNF pulsar catalog (2018 April version; Manchester et al. 2005). Our first objective is to examine the relations among three key parameters: the orbital period $P_{\mathrm{b}}$, the orbital eccentricity $\epsilon$, and the mass of the WD companion $M_{\mathrm{c}}$. For the systems with precise measurements (cf. Table 2 in Özel \& Freire 2016), we adopted their $M_{c}$ from the literature. For the others, we adopted an orbital inclination of $i=60^{\circ}$ when deriving $M_{\mathrm{c}}$ from their mass functions by assuming a neutron star mass of $1.35 M_{\odot}$. To allow a constrained analysis, we discarded the data with uncertainties $>50 \%$ in $P_{b}$ and $\epsilon$. The screening yielded a sample comprising of 58 MSP binaries with a helium (He) WD 


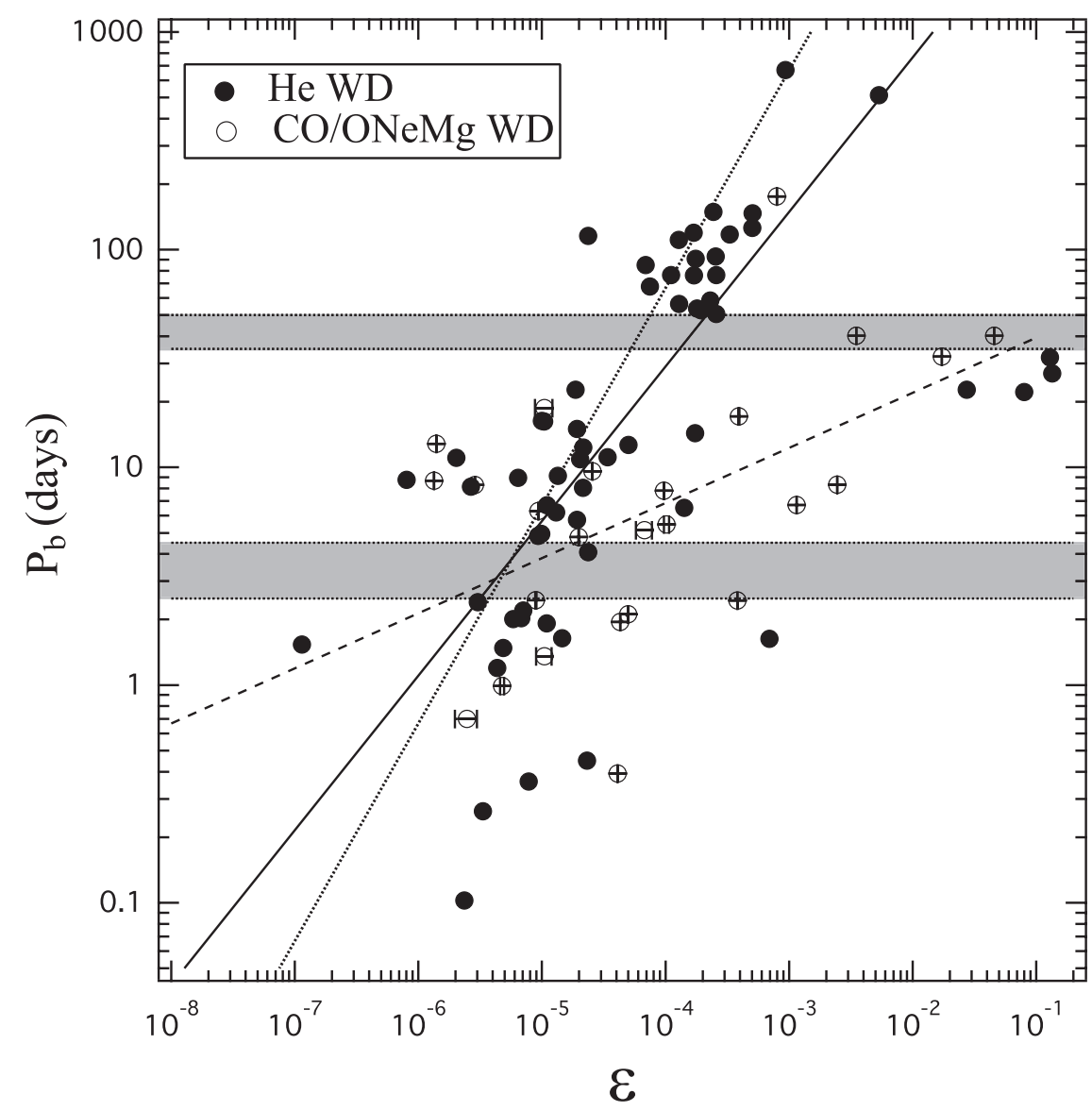

Figure 1. Orbital period $P_{\mathrm{b}}$ and eccentricity $\epsilon$ relation for MSP binaries with a He WD companion (solid symbols) and with a CO/ONeMg WD companion (open symbols). The period gaps are marked as the shaded regions. The solid and dashed lines correspond to the best linear fits between $\log P_{\mathrm{b}}$ and $\log \epsilon$ for systems containing a He WD and a CO/ONeMg WD respectively. The simple relation predicted by Phinney (1992) has also been plotted for comparison (dotted line).

companion and 25 MSP binaries with a carbon-oxygen/ oxygen-neon-magnesium $(\mathrm{CO} / \mathrm{ONeMg}) \mathrm{WD}$ companion.

We recognized that there are several recently discovered MSPs that fit our selection criteria that are not included in the aforementioned sample. PSR J2234+0611 (Antoniadis et al. 2016) and PSR J1946+3417 (Barr et al. 2017) can be found in the ATNF catalog but the nature of their companions is not specified in it. On the other hand, PSR J1618-3921 (Octau et al. 2018) is not included in the ATNF catalog. The companions of all these systems appear to be He WDs, and their eccentricities lie in the range of $\epsilon \sim 0.01-0.1$, which is higher than the general MSP population with $\mathrm{He}$ WD companions. Appending these systems to our sample, we have 61 MSP binaries with He WD companions for our analysis. The properties of all the selected MSP binaries with He WD companions and with $\mathrm{CO} / \mathrm{ONeMg}$ WD companions are summarized in Table 1 and Table 2, respectively.

\section{1. $\epsilon-P_{b}$ Relation}

Figure 1 shows the MSP binaries in the $\epsilon-P_{\mathrm{b}}$ plane. A parametric (linear correlation coefficient $r$ ) and a nonparametric (Spearman rank correlation coefficient $\rho$ ) test were applied for the correlation test between $P_{\mathrm{b}}$ and $\epsilon$, giving $r=-3.85 \times 10^{-2}$ $(p$-value $=0.77) \quad$ and $\rho=0.72 \quad\left(p\right.$-value $\left.=4.13 \times 10^{-11}\right)$, respectively, for the MSP binaries with a He WD. The apparent discrepancy between the results is caused by a group of outliers with $\epsilon>0.01$ (see Figure 1), which includes PSR J1950+2414, PSR J2234+0611，PSR J1946+3417， and PSR J1618-3921.
The eccentricity abnormality is speculated to be due to an unusual event, such as a delayed accretion-induced collapse of a massive WD during the course of the system's evolution (see Freire \& Tauris 2014). Alternatively, Antoniadis (2014) proposes that such high eccentricity could have resulted from the dynamical interaction between the binary and a circumbinary disk over $\sim 10^{4}-10^{5}$ years.

When these four eccentric binaries are excluded, the correlation analysis yields $r=0.69\left(p\right.$-value $\left.=2.94 \times 10^{-9}\right)$ and $\rho=0.74 \quad\left(p\right.$-value $\left.=4.08 \times 10^{-11}\right)$. Thus, both tests reconciled, concluding a strong $\epsilon-P_{\mathrm{b}}$ correlation. As $\rho$ is a nonparametric statistic, its estimate is therefore robust. The statistical significance it refers to would be almost unaltered when the outliers are removed. A regression analysis excluding four eccentric MSPs yielded a relation

$$
\log \left(P_{\mathrm{b}} / \text { day }\right)=(0.71 \pm 0.18) \log \epsilon+(4.30 \pm 0.81)
$$

for $P_{\mathrm{b}}$ and $\epsilon$. Here and hereafter, unless otherwise stated, the uncertainties of the parameters are of a 95\%-confidence interval that is estimated by $t_{1-\alpha / 2, \nu} \mathrm{SE}$, where $t_{1-\alpha / 2, \nu}$ is the $t$ statistic with $\alpha=0.05$ and a degree of freedom $\nu=$ (no. of data pointnumber of free parameters) and SE is the standard error of the corresponding parameters derived from the covariance matrix.

We also plotted the $\epsilon-P_{\mathrm{b}}$ relation predicted by Phinney (1992; $\epsilon \sim 1.5 \times 10^{-4}\left(P_{\mathrm{b}} / 100\right.$ days $)$; dotted line in Figure 1) for comparison. For $P_{b} \gtrsim 1$ day, our best-fit based on the current sample (i.e., Equation (1)) predicts a higher $\epsilon$ for a given $P_{b}$ than that suggested by Phinney (1992). 

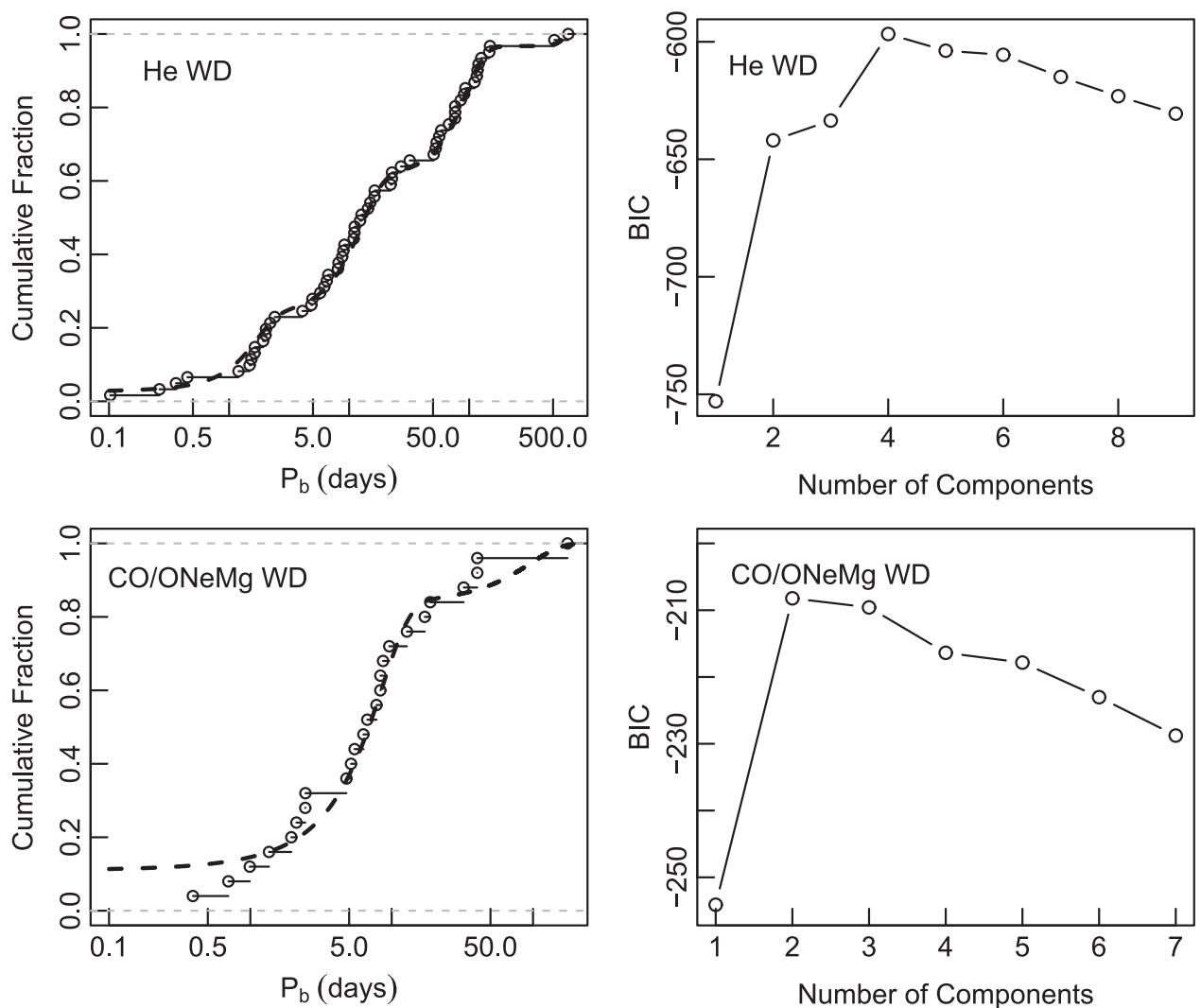

Figure 2. (Upper left panel) The unbinned empirical cumulative distribution function (CDF) of the orbital periods of MSP binaries with a He WD companion in the Galactic field (open symbols). The dashed line illustrates the four-component Gaussian mixtures model. (Upper right panel) The values of Bayesian information criterion (BIC) vs. the number of Gaussian components included in the model. (Lower left panel) Same as upper left panel but for the MSPs with CO/ONeMg WD companions. (Lower right panel) Same as upper right panel but for the MSPs with CO/ONeMg WD companions. The BIC values for eight and nine component models are undefined.

For the MSP binaries with a $\mathrm{CO} / \mathrm{ONeMg}$ (non-He) WD, there is no noticeable outlier. We obtained $r=0.19$ ( $p$-value $=$ $0.37)$ and $\rho=0.45\left(p\right.$-value $\left.=3.09 \times 10^{-2}\right)$ for the $\epsilon-P_{\mathrm{b}}$ correlation, which has a weaker significance than that of the $\mathrm{He}$ WD case. The corresponding $\epsilon-P_{\mathrm{b}}$ relation is

$$
\log \left(P_{\mathrm{b}} / \text { day }\right)=(0.25 \pm 0.18) \log \epsilon+(1.85 \pm 0.77) .
$$

The different trends in the $\epsilon-P_{\mathrm{b}}$ relations for the MSP binaries with a He WD and with a non-He WD can be seen in the bestfit relations shown in Figure 1.

\subsection{Period Gaps}

Inspecting the orbital period distribution revealed a deficiency of sources at $P_{\mathrm{b}} \sim 35-50$ days (the upper shaded region, Figure 1). Only two MSP binaries with a $\mathrm{CO} / \mathrm{ONeMg}$ WD were there, but none with a He WD. We designate this period interval as "Gap 1."

If we limit ourselves to the systems following the $\epsilon-P_{\mathrm{b}}$ relation (i.e., ignore the outlying eccentric systems), the gap size can be relaxed to $P_{\mathrm{b}} \sim 25-50$ days. This gap is known (e.g., Camilo 1995; Tauris 1996; Taam et al. 2000). For those eccentric systems, their $P_{\mathrm{b}}$ appear to be rather similar (see Figure 1). Assuming the circumbinary disk scenario, Antoniadis (2014) has simulated the distribution of $P_{\mathrm{b}}$ from 1 day to 50 days, which shows a jump of $\epsilon$ at $P_{\mathrm{b}}>10$ days. The author proposes that this might explain the existence of this period gap. At $P_{\mathrm{b}} \gtrsim 50$ days, the $\epsilon-P_{\mathrm{b}}$ correlation can be recovered due to the cessation of hydrogen flashes for proto-WDs with masses $\gtrsim 0.35 M_{\odot}$ (Antoniadis 2014).

Besides "Gap 1," we have identified another period gap, at $P_{\mathrm{b}} \sim 2.5-4.5$ days. We designate it as "Gap 2," which is highlighted by the lower shaded region in Figure 1. To examine the significance for the presence of these period gaps, we perform a model-based clustering on the distribution of $P_{\mathrm{b}}$ by using the CRAN mclust package (Fraley \& Raftery 2002, 2007).

Since the binaries with $\mathrm{He}$ and $\mathrm{CO} / \mathrm{ONeMg}$ WD companions have different evolutionary histories, we investigate their $P_{\mathrm{b}}$ separately. Assuming a mixture of Gaussian components, we carried out the maximum likelihood fits. The calculation was repeated nine times with a different number of components (1-9 Gaussians) included. The best model is chosen on the basis of Bayesian information criterion (BIC) values from the maximum likelihood estimations.

The results are shown in Figure 2. For the $P_{\mathrm{b}}$ distribution of the binaries with a $\mathrm{He} \mathrm{WD}$, the BIC indicates that four Gaussian components best fit the data (see the upper right panel of Figure 2). These four components are:

$$
\begin{cases}\mathcal{N}_{1}\left(\mu_{1}=1.38 \text { days, } \sigma_{1}=0.73 \text { days }\right), & {[0.20]} \\ \mathcal{N}_{2}\left(\mu_{2}=10.35 \text { days, } \sigma_{2}=5.75 \text { days }\right), & {[0.40]} \\ \mathcal{N}_{3}\left(\mu_{3}=80.62 \text { days, } \sigma_{3}=37.42 \text { days }\right), & {[0.37]} \\ \mathcal{N}_{4}\left(\mu_{4}=590.56 \text { days, } \sigma_{4}=78.52 \text { days }\right), & {[0.03]}\end{cases}
$$

In Equation (3), $\mu$ and $\sigma$ are the mean and standard deviation for the corresponding Gaussian components. And the numbers 


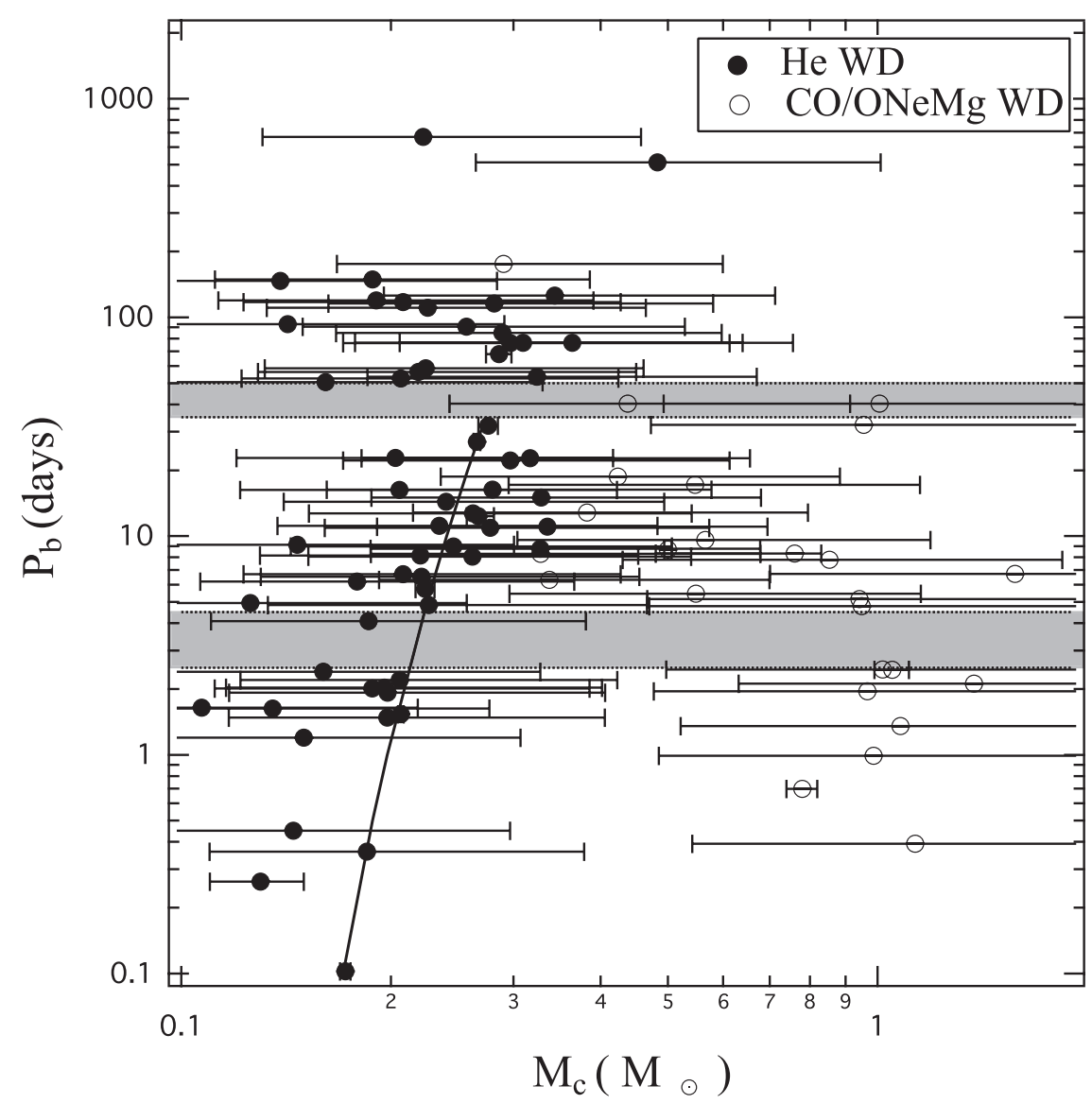

Figure 3. Orbital period $P_{\mathrm{b}}$ and companion mass $M_{\mathrm{c}}$ relation for MSP binaries with a He WD companion (solid symbols) and with a CO/ONeMg companion (open symbols). For those have been listed in the Table 2 of Özel \& Freire (2016), which have dedicated measurements of $M_{\mathrm{c}}$, their values and the uncertainties are adopted. For the others, $M_{\mathrm{c}}$ are estimated from their mass functions by assuming an orbital inclination $i=60^{\circ}$ and $M_{\mathrm{NS}}=1.35 M_{\odot}$. Their error bars are estimated by varying $i$ from $18^{\circ}$ to $90^{\circ}$. The period gaps are marked as the shaded regions. The solid line illustrates the best-fit $M_{\mathrm{c}}-P_{\mathrm{b}}$ relation for the LB of systems with a He WD companion.

in the square brackets are the fraction of the data covered by that component. The upper left panel of Figure 2 show the empirical cumulative distribution function $(\mathrm{CDF})$ of the data for the $P_{\mathrm{b}}$ distribution of the binaries with a He WD (open symbols). Gap 1 and Gap 2 appears as the flattened regions in the CDF. The dashed curve is the four-component Gaussian mixture model. It appears that the model can reasonably describe the data.

We further check if the separations among these components are significant by computing the Ashman's $D$ statistic (Ashman et al. 1994). All the pairs result in $D>12$, which indicates clear separations among them.

Considering the clusters $\mathcal{N}_{1}$ and $\mathcal{N}_{2}$, the $1 \sigma$ upper-bound of $\mathcal{N}_{1}$ and the $1 \sigma$ lower-bound of $\mathcal{N}_{2}$ span a range of $\sim 2.1-4.6$ days, which encompasses Gap 2. Similarly, the clusters $\mathcal{N}_{2}$ and $\mathcal{N}_{3}$ are found to encompass Gap 1. Based on the aforementioned analysis, the presence of these two period gaps are siginificant.

Although the BIC suggests that the presence of $\mathcal{N}_{4}$ is also significant, this component only consists of two objects, namely PSR J0407+1607 $\left(P_{\mathrm{b}}=669.07\right.$ days $)$ and PSR J0214 +5222 $\left(P_{\mathrm{b}}=512.04\right.$ days $)$. With such limited samples, we cannot draw a firm conclusion regarding its existence and this component will not be considered in all subsequent analyses.

We apply the same analysis to the MSP binaries with $\mathrm{CO} /$ $\mathrm{ONeMg}$ WD companions. The values of the BIC (lower right panel of Figure 2) indicate that this population consists of two Gaussian components:

$$
\left\{\begin{array}{ll}
\mathcal{N}_{1}^{\prime}\left(\mu_{1}=5.95 \text { days, } \sigma_{1}=4.67 \text { days }\right), & {[0.80]} \\
\mathcal{N}_{2}^{\prime}\left(\mu_{2}=61.04 \text { days, } \sigma_{2}=58.55 \text { days }\right), & {[0.20]}
\end{array} .\right.
$$

Comparing this model to the CDF constructed from the data (lower left panel of Figure 2), there are discrepancies between the model and the data at $P_{\mathrm{b}} \lesssim 5$ days and $P_{\mathrm{b}} \gtrsim 20$ days. The poor fit can be ascribed to the small sample of data. Although the analysis suggests that this population might contain more than a single component, the location of both Gap 1 and Gap 2 cannot be constrained solely with the current MSP population with $\mathrm{CO} / \mathrm{ONeMg} \mathrm{WD}$ companions.

\section{3. $M_{c}-P_{b}$ Relation}

Figure 3 shows the $M_{\mathrm{c}}-P_{\mathrm{b}}$ distributions of the systems. Since their mass estimates adopted in this work are derived from the mass functions by assuming $M_{\mathrm{NS}}=1.35 M_{\odot}$ and $i=60^{\circ}$, there is no error estimate provided by the ATNF catalog. For those sources with precise mass estimates, we adopted their values and the uncertainties given in Özel \& Freire (2016). For the others, the uncertainties of $M_{\mathrm{c}}$ are difficult to estimate. This can be ascribed to two facts: (1) the distribution of $i$ is likely to be uniform, which leaves $M_{\mathrm{c}}$ unconstrained without the measurement of $i$; (2) $M_{\mathrm{NS}}$ for MSPs can be different from 
the canonical value because of the accretion processes. Following van Kerkwijk et al. (2005), we reflect on the uncertainties of $M_{c}$ by varying $i$ from $18^{\circ}$ to $90^{\circ}$ for those do not have dedicated mass measurement. The results are shown in Figure 3.

Interestingly, MSP binaries with a He WD are segregated into two subpopulations by Gap 1 whereas the MSP binaries with a non-He WD companion are divided into two subpopulations by Gap 2. For the MSP binaries containing a He WD above Gap 1 (the long-period systems), a correlation analysis of $P_{\mathrm{b}}$ and $M_{\mathrm{c}}$ gave $r=0.26(p$-value $=0.25)$ and $\rho=-0.05$ ( $p$-value $=0.84)$, implying that $P_{\mathrm{b}}$ and $M_{\mathrm{c}}$ have no strong dependence. We refer to this subpopulation as the "horizontal branch" (hereafter HB) of the MSP binaries with a He WD companion. On the contrary, for the binaries below Gap 1 (the short-period systems) there is a strong correlation between $P_{\mathrm{b}}$ and $M_{\mathrm{c}}$, with $r=0.64\left(p\right.$-value $\left.=6.74 \times 10^{-6}\right)$ and $\rho=0.74 \quad\left(p\right.$-value $\left.=4.37 \times 10^{-8}\right)$. We refer to this subpopulation as the "lower branch" (hereafter LB) of the MSP binaries with a He WD companion. We note that all the eccentric systems follow the general trend of the LB. This is consistent with the prediction by Antoniadis (2014).

Tauris \& Savonije (1999) have computed the correlation between $M_{\mathrm{c}}$ and $P_{\mathrm{b}}$ numerically. They found that their model calculations can be fitted to a form of

$$
\frac{M_{\mathrm{c}}}{M_{\odot}}=\left(\frac{P_{\mathrm{b}}}{b}\right)^{1 / a}+c,
$$

where $(a, b, c)$ depend on the composition of the donor and $P_{\mathrm{b}}$ in units of days. Fitting Equation (5) to the LB of the MSP binaries with a He WD companion in our sample yields a set of parameters $a=4.91 \pm 2.26, b=(4.18 \pm 13.1) \times 10^{5}$, and $c=0.12 \pm 0.04$. The results are comparable with Tauris \& Savonije (1999; see Equation (21) in their paper). The best-fit $M_{\mathrm{c}}-P_{\mathrm{b}}$ relation is illustrated by the solid line in Figure 3.

From the data, we deduced that $(34 \pm 12) \%$ of MSP binaries having a $\mathrm{He}$ WD companion would be in the $\mathrm{HB}$ and $(66 \pm 12) \%$ in the LB. The $95 \%$ confidence intervals were computed by the standard maximum likelihood Wald estimator.

The binaries with a $\mathrm{CO} / \mathrm{ONeMg} \mathrm{WD}$ also show a HB (longperiod systems) and a LB (short-period systems) bisected by Gap 2 in the $M_{\mathrm{c}}-P_{\mathrm{b}}$ plane, with also a turnover between the two branches. In comparison with the systems with a He WD, they as a group are shifted to the lower right corner of the $M_{\mathrm{c}}-P_{\mathrm{b}}$ plane. This is partly because $\mathrm{CO} / \mathrm{ONeMg}$ WDs are generally more massive. It is, however, puzzling that systems with a $\mathrm{CO} /$ ONeMg WD tend to have shorter orbital periods than the systems with a He WD. We deduced that there are $(68 \pm 18) \%$ and $(32 \pm 18) \%$ MSP binaries containing a CO/ONeMg WD in their $\mathrm{HB}$ and the $\mathrm{LB}$ respectively. No significant correlation was found between $P_{\mathrm{b}}$ and $M_{\mathrm{c}}$ for both the populations above and below Gap 2.

To examine the mass distributions of the WDs in the MSP binaries we conducted two-sample Kolmogorov-Smirnov (KS) and Anderson-Darling (AD) tests for the following samples: (a) WDs in all MSP binaries, (b) He WDs in MSP binaries, and (c) non-He WDs in MSP binaries, The SDSS field WDs in the Montreal WD Database (Dufour et al. 2017; designated as (x)). For the $(x-a),(x-b)$, and $(b-c)$ comparisons, we obtained $p$-values $\ll 10^{-12}$ in both $\mathrm{KS}$ and $\mathrm{AD}$ tests; for the $(\mathrm{x}-\mathrm{c})$ comparison, $p$-values of $1.1 \times 10^{-4}$ in the $\mathrm{KS}$ test and
$2.2 \times 10^{-7}$ in the $\mathrm{AD}$ test. A Gaussian fit to the mass distribution of the SDSS WDs gave $\left(M_{\mathrm{wd}}, \sigma\right)=(0.622,0.157)$ (in units of $\left.M_{\odot}\right)$ while WDs in the MSP binaries gave $\left(M_{\mathrm{c}}\right.$, $\sigma)=(0.394,0.319)$. When MSP binaries were separated into the HB and LB subpopulations, we obtained $\left(M_{\mathrm{c}}, \sigma\right)=(0.231$, $0.070)[\mathrm{HB}+\mathrm{LB}],(0.255,0.083)[\mathrm{HB}]$ and $(0.218,0.059)$ [LB] for the systems with a He WD and $\left(M_{\mathrm{c}}, \sigma\right)=(0.792,0.342)$ $[\mathrm{HB}+\mathrm{LB}],(0.671,0.340)[\mathrm{HB}]$, and $(1.049,0.168)[\mathrm{LB}]$ for the systems with a $\mathrm{CO} / \mathrm{ONeMg} \mathrm{WD}$.

\section{Discussions and Conclusions}

In summary, our analyses have shown the following:

(i) The orbital periods $P_{\mathrm{b}}$ of the systems are not uniformly distributed, with two gaps located at $P_{\mathrm{b}} \sim 35-50$ days (Gap 1) and 2.5-4.5 days (Gap 2). Gap 1 divides the MSP binaries with a He WD into two distinctive subgroups, the $\mathrm{HB}$ and LB, and similarly Gap 2 separates the MSP binaries with a $\mathrm{CO} / \mathrm{ONeMg} \mathrm{WD}$ into the $\mathrm{HB}$ and $\mathrm{LB}$ subgroups.

(ii) Both MSP binaries with a He WD and MSP binaries with a $\mathrm{CO} / \mathrm{ONeMg}$ WD show a positive correlation between $P_{\mathrm{b}}$ and $\epsilon$. Their $\epsilon-P_{\mathrm{b}}$ relations are, however, not identical.

(iii) Neither MSP binaries with a He WD nor those with a non-He WD show $P_{\mathrm{b}}-M_{\mathrm{c}}$ dependence on the HB (above the period gap).

(iv) For the MSP binaries with a He WD, $P_{\mathrm{b}}$ and $M_{\mathrm{c}}$ appear correlated in the LB (below Gap 1). Such a correlation is not present in the LB of the MSP binaries with a $\mathrm{CO} /$ ONeMg WD.

These phenomena are a consequence of the orbital evolutionary dynamics of the MSP-WD binaries, which manifests as migration flows in the $M_{\mathrm{c}}-\xi$ plane (Figure 4). The variable $\xi \equiv R_{\mathrm{c}}{ }^{2} / 4 a^{2} M_{\mathrm{c}}$, where $R_{\mathrm{c}}$ is the radius of the WD companion and $a$ is the orbital separation of the system, measures the amount of pulsar wind that could be intercepted by the WD, per unit WD mass, and hence is an indicator of WD mass loss under pulsar-wind ablation. In the $M_{\mathrm{c}}-\xi$ plane, the rate of change in the system's orbital period are vectors normal to the constant $P_{\mathrm{b}}$ contours. The rate of change in the WD companion's mass are horizontal vectors with a negative direction. Adding these two vectors gives the individual migration velocity of the source, whose horizontal component is always negative.

The migration flow is driven by the angular momentum loss from the orbit and the mass loss from the companion star caused by the interactions between the pulsar and the companion. The time derivative of Kepler's law gives the orbital evolutionary equation (with component stars of masses $m_{1}$ and $\left.m_{2}\right)$ :

$$
\begin{aligned}
\frac{\dot{J}}{J}= & \frac{\dot{m}_{1}}{m_{1}}\left[\frac{2+3 q}{3(1+q)}\right]+\frac{\dot{m}_{2}}{m_{2}}\left[\frac{3+2 q}{3(1+q)}\right] \\
& +\frac{\dot{P}_{\mathrm{b}}}{P_{\mathrm{b}}}\left[\frac{1}{3}\right]-\frac{\dot{\epsilon}}{\epsilon}\left[\frac{\epsilon^{2}}{\left(1-\epsilon^{2}\right)}\right],
\end{aligned}
$$

where $q=m_{2} / m_{1}, J$ is the orbital angular momentum, and "." denotes the time derivative. By setting $\epsilon \approx 0$ (almost circularized orbit), $m_{2}=M_{\mathrm{c}}, \dot{m}_{1}=\dot{M}_{\mathrm{MSP}} \approx 0$ (insignificant mass gain), and $f(q)=(3+2 q) / 3(1+q)$ and introducing a parameterization $(\dot{J} / J) /\left(\dot{M}_{\mathrm{c}} / M_{\mathrm{c}}\right)=\alpha\left(X_{\mathrm{i}}, P, q\right)>0$, where 


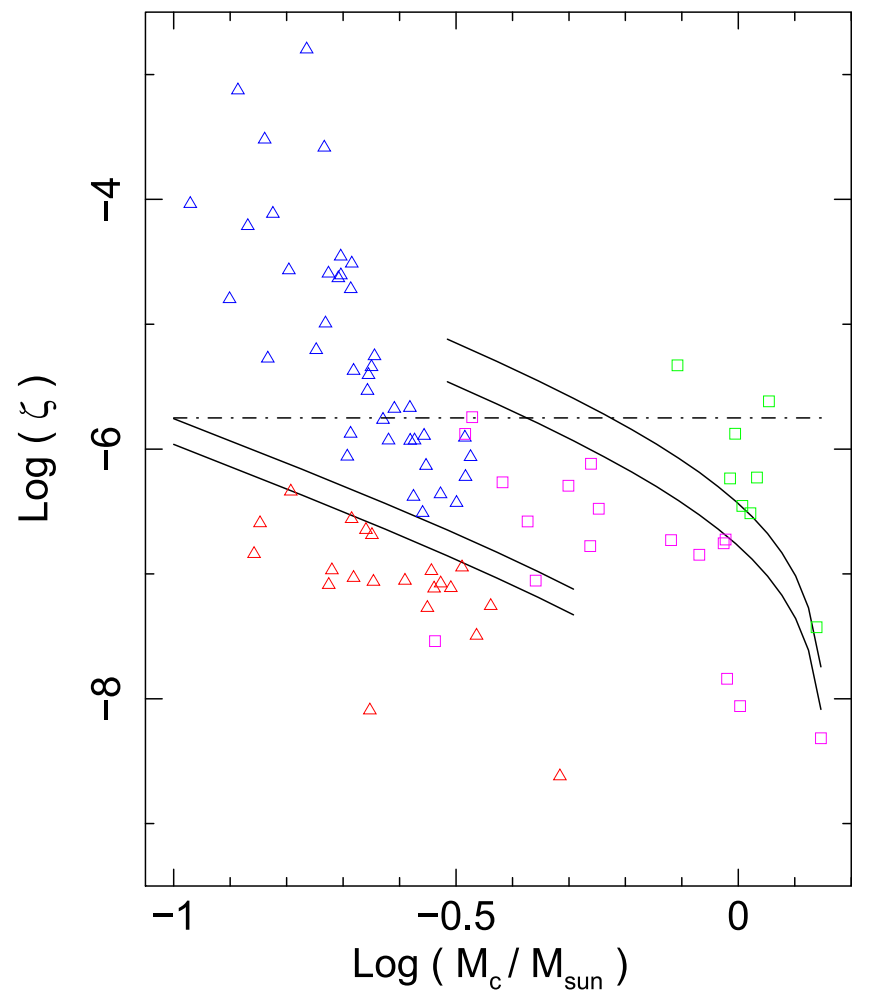

Figure 4. $M_{\mathrm{c}}-\xi$ relation for the MSP-WD binaries, where $\xi\left(\equiv R_{\mathrm{c}}{ }^{2} / 4 a^{2} M_{\mathrm{c}}\right)$ is a measure of maximum amount of pulsar wind that the WD can intercept. The black curves are the period contours marking the two periods' gaps, with Gap 1 on the left side and Gap 2 on the right side in the $M_{\mathrm{c}}-\xi$ plane. The orbital periods corresponding to the contours from top to bottom are therefore $P_{\mathrm{b}} /$ day $=2.5,4.5,35$, and 50. MSP binaries with a He WD are represented by triangles (red for HB systems and blue for LB systems), and MSP binaries with a $\mathrm{CO} / \mathrm{ONeMg} \mathrm{WD}$ by squares (violet for $\mathrm{HB}$ systems and green for $\mathrm{LB}$ systems). The dashed line is a schematic approximate reference, above which pulsar-wind ablation is able to cause significant mass loss from the WD. Orbital period evolves along the normals to the period contours, and WD mass loss progresses along the horizontal axis. The ratio of the magnitude of the former to that of the latter is determined by $3[\alpha-f(q)]$ (see the text). In our calculations, the MSPs are neutron stars with $1.35-M_{\odot}$, and the WDs have radii given by the Nauenberg (1972) relation.

$X_{\mathrm{i}}$ are variables intrinsic to the MSP, we obtained the equation for period change in response to the companion's mass loss:

$$
\frac{\dot{P}}{P} \approx \frac{\dot{M}_{\mathrm{c}}}{M_{\mathrm{c}}}[3(\alpha-f(q))] .
$$

Note that $f(q)$ is a slowly varying function, with $f(q) \approx 0.977$ for $\left(M_{\mathrm{MSP}}, M_{\mathrm{c}}\right)=\left(1.35 M_{\odot}, 0.1 M_{\odot}\right)$ and $f(q) \approx 0.830$ for $\left(M_{\mathrm{MSP}}, M_{\mathrm{c}}\right)=\left(1.35 M_{\odot}, 1.4 M_{\odot}\right)$.

We propose that the period gaps were developed when the progenitors of the MSP-WD binaries were in the transition from being a mass-transfer system to an MSP system. By the time of this transition, the companion star's progenitor would have already shredded most of it mass, implying the condition $q<1$ had be satisfied. This prevents a runaway mass transfer process and halts a rapid spiraling-in. If only mass exchange between the component stars occurs, $\dot{J} \approx 0$ on the dynamical timescale. Hence $\alpha \approx 0$ for the binary at this stage, when the mass transfer operates. The binary's orbit would expand in response to the companion's mass loss. As such, the system migrates leftward and downward across the $M_{\mathrm{c}}-\xi$ plane. Pulsarwind ablation will onset when the accretion pauses. The mass outflow from WD induced by the pulsar wind and the viscous drag of the ablated material on the WD orbital motion facilitates the angular-momentum extraction from the orbit. When the angular momentum loss is efficient, i.e., $\alpha \gg 1$, mass loss from the companion will shorten the orbital period, and the system will migrate leftward and upward across the $M_{\mathrm{c}}-\xi$ plane. The period gaps are the separatrices that divide the upward and downward migration tracks of the sources during the accretion-MSP transition.

(i) Locations of the two period gaps: The progenitors of $\mathrm{CO} /$ ONeMg WDs were able to evolve through the asymptotic giant branch and reach the horizontal giant branch, and they are relatively massive (about $3 M_{\odot}$ or higher). The MSP binaries with a $\mathrm{CO} / \mathrm{ONeNgWDs}$ must have survived the complete spiraling-in during the temperature oscillation phase of the companion star when $\mathrm{C}$ burning proceeds to $\mathrm{O}$ burning, and the second common envelope phase (if present). The maximum size of the companion star is constrained by the orbital separation $a$, and hence in the final mass-transfer episodes constrained by the period gap in our proposed scenario. With $a=4.65 R_{\odot}$ $\left(P_{\mathrm{b}} / \text { day }\right)^{2 / 3}(1+q)^{1 / 3}, P_{\mathrm{b}} \sim(2.5-4.5)$ day (Gap 2) corresponds to $a \sim(8.6-13)(1+q)^{1 / 3} R_{\odot}$. The radius of an evolved star with a $3-M_{\odot}$ main-sequence progenitor in the $\mathrm{C} / \mathrm{O}$ burning stage will reach above $10 R_{\odot}$ (see Maeder \& Meynet 1989), consistent with the period-gap formation scenario (for Gap 2) that we propose, if nuclear evolution drives the final episodes of the mass transfer process. He WDs have less massive main-sequence progenitors (about $1 M_{\odot}$ or lower). When the companion star of the progenitor MSP binary evolves into the He burning red giant stage, it expands substantially. A common envelope could be avoided if the two stars have a sufficiently large orbital separation. For these systems, mass transfer is expected to operate in a somewhat steady manner, as the companion star is less massive than the neutron star. This leads to orbital expansion and period lengthening. $P_{\mathrm{b}} \sim(35-50)$ day (Gap 1) corresponds to $a \sim(50-63)(1+q)^{1 / 3} R_{\odot}$. The radius of a star, starting as the $1-M_{\odot}$ main-sequence star, is $\sim 30 R_{\odot}$ at the end of its He burning (Charbonnel et al. 1999), which $\sim 0.5$ times of the orbital separation inferred from Gap 1, a configuration where Roche-lobe filling mass-transfer is possible (see, e.g., Eggleton 1983).

(ii) $\mathrm{HB}$ formation: We interpret that the $\mathrm{HB}$ is a piling-up of systems, caused by orbital expansion in the final mass-transfer episodes when the progenitor binaries were at the transition from being an accretion system to an MSP system. The lack of strong dependence of $M_{\mathrm{c}}$ for both MSP binaries with a He WD and with a non-He WD is a consequence of the combination of the following: (i) a weak dependence of $f(q)$ on $q$, which gives $\dot{P}_{\mathrm{b}} / \dot{M}_{\mathrm{c}} \approx-0.9\left(P_{\mathrm{b}} / M_{\mathrm{c}}\right)$, and (ii) that the constant $P_{\mathrm{b}}$ contours are almost straight lines in the $M_{\mathrm{c}}-\xi$ plane spanning from $\log \left(M_{\mathrm{c}} / M_{\odot}\right)=-1.0$ to $\log \left(M_{\mathrm{c}} / M_{\odot}\right) \approx 0.0$. As such, the systems have a fairly uniform velocity over a wide $M_{\mathrm{c}}$ range when migrating away from their respective period gaps in the $M_{\mathrm{c}}-\xi$ plane on their course to becoming "full-fledged" MSP binaries. Although the systems would eventually evolve across the period gap later as MSP-WD binaries, the process will be slow, as at the HB the amount of pulsar wind intercepted by the WD is low (see Figure 4). Without a strong outflow from the WD, direct extraction of angular momentum from the binary's orbit cannot be efficient. Moreover, there will be no viscous drag on the WD's motion when ambient material is absent. When $\alpha$ could not attain a high value, the MSP-WD binaries will linger in the HBs. 
(iii) LB morphologies: The pattern formation in the $M_{\mathrm{c}}-P_{\mathrm{b}}$ plot (Figure 2) is simply a reflection of the migration of the MSP binaries in the $M_{\mathrm{c}}-\xi$ plane (Figure 4), which is driven by pulsar-wind ablation of the WD. The morphology of the LB of the MSP binaries with a He WD is caused by the flow confluence of systems of all masses in the $M_{\mathrm{c}}-\xi$ plane, in particular, the rapid orbital evolution of the systems with a very low-mass WD $\left(\sim 0.1 M_{\odot}\right)$. Low-mass WDs have large radii. In addition to their efficient interception of pulsar wind, they are subject to large viscous drag if ambient material is present. For the lower WD-mass MSP binaries, a large value for $\alpha$ can be attained, implying a large $\left|\dot{P}_{\mathrm{b}} / P_{\mathrm{b}}\right|$ to $\left|\dot{M}_{\mathrm{c}} / M_{\mathrm{c}}\right|$ ratio. Thus, they have larger upward migration velocity components in the $M_{\mathrm{c}}-\xi$ plane than their higher WD-mass counterparts. The confluence flow and the $M_{\mathrm{c}}$ dependent migration explain the LB tilting for the MSP binaries with a He WD and the apparent larger spread of $\xi$ in the LB at the low WD-mass end. Such a pattern is, however, not expected for the LB of the MSP binaries with a $\mathrm{CO} / \mathrm{ONeMg} \mathrm{WD}$. The pulsar-wind ablation of their WD is not efficient enough to drive rapid migration across the $M_{\mathrm{c}}-\xi$ plane. At the high WD-mass end, the bending of the constant $P_{\mathrm{b}}$ contours causes the $\dot{P}_{\mathrm{b}} / P_{\mathrm{b}}$ to have a strong horizontal projection opposite to the direction of $\dot{M}_{\mathrm{c}} / M_{\mathrm{c}}$. Therefore, the massive-WD MSP systems can only migrate upward slowly in the $M_{\mathrm{c}}-\xi$ plane. Instead, they slide gradually and only slightly deviate from the tangents of the constant $P_{\text {orb }}$ contours. Only for systems with a WD of $\approx 1 M_{\odot}$ or lower, such "confinement" to the migration flow becomes inefficient. Note that the MSP binaries with a low-mass $\mathrm{CO} / \mathrm{ONeMg}$ WD in their $\mathrm{HB}$ and the MSP binaries with a high-mass He WD in their LB have very similar orbital periods, pulsar-wind ablation efficiencies and WD masses, and hence are subject to similar viscous drag. In the $M_{\mathrm{c}}-\xi$ plane, MSP binaries with a low-mass
$\mathrm{CO} / \mathrm{ONeMg}$ WD in the HB would therefore join the confluent flows of the MSP binaries with a He WD in LB instead of migrating cross their own period gap, Gap 2 (see Figure 4).

In summary, we attributed the period gaps and their locations to the conditions of the latest stages of stellar evolution of the WD progenitor. The evolutionary bifurcation of the MSP binaries with a He WD in the $\mathrm{HB}$ and $\mathrm{LB}$ is due to relative efficiencies of angular momentum loss induced by the pulsarwind ablation of the WD, which naturally gives a positive correlation between $P_{\mathrm{b}}$ and $M_{\mathrm{c}}$ in the LB systems. The MSP binaries with a low-mass $\mathrm{CO} / \mathrm{ONeMg} \mathrm{WD}$ in the $\mathrm{HB}$ have similar pulsar-wind ablation efficiencies as the MSP binaries with a high-mass He WD in the LB, and hence these binaries migrate similarly in the $M_{\mathrm{c}}-\xi$ plane. The MSP binaries with a massive $\mathrm{CO} / \mathrm{ONeMg} \mathrm{WD}\left(M_{\mathrm{c}} \gtrsim 1 M_{\odot}\right)$ linger in the vicinity of their birth places because the amount of pulsar wind intercepted by the WD is insufficient to drive a rapid orbital evolution.

C.Y.H. is supported by the National Research Foundation of Korea grant 2016R1A5A1013277; A.K.H.K. by the Ministry of Science and Technology of Taiwan grants 1062918-I-007-005 and 105-2112-M-007-033-MY2; P.H.T.T. by the SYSU One Hundred Talents Program; and Q.H. by a NJU School of Astronomy and Space Sciences Overseas Research Scholarship and a UCL-MSSL Summer Research Studentship.

\section{Appendix}

The data used for this investigation are given in Table 1 and Table 2. 
Table 1

Orbital Properties of MSP Binaries with He WD Companions

\begin{tabular}{|c|c|c|c|}
\hline Pulsar Name ${ }^{\mathrm{a}}$ & $\begin{array}{l}\text { Orbital Period } P_{\mathrm{b}} \\
\text { (days) }\end{array}$ & $\begin{array}{c}\text { Eccentricity } \epsilon \\
\left(10^{-6}\right)\end{array}$ & $\begin{array}{c}\text { Companion Mass } M_{\mathrm{c}} \\
\left(M_{\odot}\right)\end{array}$ \\
\hline $\mathrm{J} 0348+0432$ & $0.1024\left( \pm 7 \times 10^{-12}\right)$ & $2.36( \pm 1.0)$ & $0.172( \pm 0.003)$ \\
\hline $\mathrm{J} 0751+1807$ & $0.2631\left( \pm 7 \times 10^{-12}\right)$ & $3.322( \pm 0.5)$ & $0.13( \pm 0.02)$ \\
\hline $\mathrm{J} 1816+4510$ & $0.3609\left( \pm 2 \times 10^{-10}\right)$ & $7.810( \pm 2.0)$ & $0.185(-0.075,+0.194)$ \\
\hline $\mathrm{J} 1431-4715$ & $0.4497\left( \pm 7 \times 10^{-10}\right)$ & $23.19( \pm 0.8)$ & $0.145(-0.058,+0.152)$ \\
\hline J0613-0200 & $1.1985\left( \pm 1.4 \times 10^{-11}\right)$ & $4.350( \pm 0.3)$ & $0.150(-0.060,+0.157)$ \\
\hline $\mathrm{J} 2043+1711$ & $1.4823\left( \pm 1.5 \times 10^{-11}\right)$ & $4.868( \pm 0.07)$ & $0.198(-0.081,+0.208)$ \\
\hline J1909-3744 & $1.5334\left( \pm 1.3 \times 10^{-11}\right)$ & $0.1140( \pm 0.01)$ & $0.2067( \pm 0.0019)$ \\
\hline $\mathrm{J} 0337+1715$ & $1.6294\left( \pm 5 \times 10^{-9}\right)$ & $691.8( \pm 0.2)$ & $0.135(-0.054,+0.142)$ \\
\hline $\mathrm{J} 1622-6617$ & $1.6406\left( \pm 8 \times 10^{-9}\right)$ & $14.56( \pm 0.012)$ & $0.107(-0.042,+0.112)$ \\
\hline J1514-4946 & $1.9227\left( \pm 5 \times 10^{-9}\right)$ & $10.90( \pm 0.003)$ & $0.198(-0.081,+0.208)$ \\
\hline J1902-5105 & $2.0118\left( \pm 9 \times 10^{-10}\right)$ & $5.864\left( \pm 8 \times 10^{-7}\right)$ & $0.188(-0.076,+0.197)$ \\
\hline $\mathrm{J} 0218+4232$ & $2.0288\left( \pm 9 \times 10^{-11}\right)$ & $6.801( \pm 0.4)$ & $0.196(-0.080,+0.206)$ \\
\hline $\mathrm{J} 2017+0603$ & $2.1985\left( \pm 1.2 \times 10^{-10}\right)$ & $7.060( \pm 0.09)$ & $0.206(-0.084,+0.217)$ \\
\hline $\mathrm{J} 1901+0300$ & $2.3992\left( \pm 6 \times 10^{-9}\right)$ & $3.027( \pm 1.0)$ & $0.160(-0.064,+0.168)$ \\
\hline $\mathrm{J} 1045-4509$ & $4.0835\left( \pm 3 \times 10^{-10}\right)$ & $23.67( \pm 0.017)$ & $0.186(-0.075,+0.195)$ \\
\hline $\mathrm{J} 0557+1550$ & $4.8466\left( \pm 4 \times 10^{-9}\right)$ & $9.3( \pm 0.4)$ & $0.227(-0.094,+0.240)$ \\
\hline $\mathrm{J} 1745-0952$ & $4.9435\left( \pm 1.2 \times 10^{-8}\right)$ & $9.849( \pm 2.8)$ & $0.126(-0.050,+0.131)$ \\
\hline J0437-4715 & $5.7410\left( \pm 4 \times 10^{-7}\right)$ & $19.18( \pm 0.0015)$ & $0.224( \pm 0.007)$ \\
\hline $\mathrm{J} 1545-4550$ & $6.2031\left( \pm 8 \times 10^{-9}\right)$ & $13.00( \pm 0.4)$ & $0.179(-0.072,+0.188)$ \\
\hline $\mathrm{J} 1017-7156$ & $6.5119\left( \pm 2 \times 10^{-6}\right)$ & $142.0( \pm 0.02)$ & $0.221(-0.091,+0.234)$ \\
\hline $\mathrm{J} 1835-0114$ & $6.6925\left( \pm 4 \times 10^{-7}\right)$ & $11.00( \pm 3.0)$ & $0.208(-0.085,+0.220)$ \\
\hline J1543-5149 & $8.0608\left( \pm 9 \times 10^{-9}\right)$ & $21.46( \pm 0.06)$ & $0.262(-0.110,+0.278)$ \\
\hline $\mathrm{J} 1813-2621$ & $8.1598\left( \pm 1 \times 10^{-8}\right)$ & $2.657( \pm 1.0)$ & $0.220(-0.091,+0.233)$ \\
\hline $\mathrm{J} 1125-6014$ & $8.7526\left( \pm 5 \times 10^{-8}\right)$ & $0.8016( \pm 0.15)$ & $0.328(-0.141,+0.351)$ \\
\hline $\mathrm{J} 1405-4656$ & $8.9564\left( \pm 7 \times 10^{-8}\right)$ & $6.403( \pm 2.5)$ & $0.246(-0.102,+0.260)$ \\
\hline $\mathrm{J} 1056-7117$ & $9.1388\left( \pm 5 \times 10^{-7}\right)$ & $13.42( \pm 4.0)$ & $0.147(-0.059,+0.154)$ \\
\hline J1918-0642 & $10.9132\left( \pm 1.6 \times 10^{-10}\right)$ & $20.34( \pm 1.5)$ & $0.278(-0.117,+0.295)$ \\
\hline $\mathrm{J} 1903-7051$ & $11.0508\left( \pm 2 \times 10^{-8}\right)$ & $2.030( \pm 0.005)$ & $0.336(-0.144,+0.359)$ \\
\hline $\mathrm{J} 1804-2717$ & $11.1287\left( \pm 3 \times 10^{-9}\right)$ & $34.06( \pm 0.16)$ & $0.235(-0.097,+0.248)$ \\
\hline $\mathrm{J} 1857+0943$ & $12.3272\left( \pm 1.8 \times 10^{-10}\right)$ & $21.64( \pm 0.03)$ & $0.267(-0.010,+0.014)$ \\
\hline $\mathrm{J} 2236-5527$ & $12.6892\left( \pm 1.4 \times 10^{-7}\right)$ & $50.20( \pm 1.8)$ & $0.262(-0.110,+0.278)$ \\
\hline $\mathrm{J} 1600-3053$ & $14.3485\left( \pm 3 \times 10^{-6}\right)$ & $173.7( \pm 0.009)$ & $0.240(-0.100,+0.254)$ \\
\hline $\mathrm{J} 1810-2005$ & $15.0120\left( \pm 4 \times 10^{-8}\right)$ & $19.24( \pm 0.003)$ & $0.329(-0.141,+0.352)$ \\
\hline $\mathrm{J} 1938+2012$ & $16.2558\left( \pm 1 \times 10^{-7}\right)$ & $10.40( \pm 0.9)$ & $0.206(-0.084,+0.217)$ \\
\hline $\mathrm{J} 1741+1351$ & $16.3353\left( \pm 5 \times 10^{-10}\right)$ & $9.984( \pm 0.16)$ & $0.280(-0.118,+0.298)$ \\
\hline $\mathrm{J} 1950+2414$ & $22.1914\left( \pm 1 \times 10^{-6}\right)$ & $7.981 \times 10^{4}( \pm 0.12)$ & $0.297(-0.126,+0.316)$ \\
\hline $\mathrm{J} 1709+2313$ & $22.7119\left( \pm 2 \times 10^{-8}\right)$ & $18.70( \pm 0.2)$ & $0.317(-0.136,+0.338)$ \\
\hline $\mathrm{J} 1844+0115$ & $50.6459\left( \pm 1.1 \times 10^{-6}\right)$ & $257.8( \pm 1.2)$ & $0.161(-0.065,+0.169)$ \\
\hline $\mathrm{J} 1825-0319$ & $52.6305\left( \pm 1.6 \times 10^{-6}\right)$ & $193.9( \pm 1.2)$ & $0.207(-0.085,+0.218)$ \\
\hline J0614-3329 & $53.5846\left( \pm 8 \times 10^{-7}\right)$ & $180.1( \pm 0.1)$ & $0.324(-0.139,+0.347)$ \\
\hline $\mathrm{J} 2033+1734$ & $56.3078\left( \pm 7 \times 10^{-8}\right)$ & $128.7( \pm 0.05)$ & $0.219(-0.090,+0.231)$ \\
\hline $\mathrm{J} 1910+1256$ & $58.4667\left( \pm 8 \times 10^{-9}\right)$ & $230.2( \pm 0.018)$ & $0.225(-0.093,+0.237)$ \\
\hline $\mathrm{J} 1713+0747$ & $67.8251\left( \pm 1.6 \times 10^{-9}\right)$ & $74.94( \pm 0.0006)$ & $0.286( \pm 0.012)$ \\
\hline $\mathrm{J} 1455-3330$ & $76.1746\left( \pm 1.1 \times 10^{-8}\right)$ & $169.6( \pm 0.013)$ & $0.297(-0.126,+0.316)$ \\
\hline $\mathrm{J} 1125-5825$ & $76.4032\left( \pm 5 \times 10^{-8}\right)$ & $257.2( \pm 0.03)$ & $0.310(-0.132,+0.330)$ \\
\hline $\mathrm{J} 2019+2425$ & $76.5116\left( \pm 2 \times 10^{-8}\right)$ & $111.1( \pm 0.04)$ & $0.364(-0.158,+0.392)$ \\
\hline $\mathrm{J} 1850+0124$ & $84.9499\left( \pm 4 \times 10^{-6}\right)$ & $69.00( \pm 1.3)$ & $0.289(-0.122,+0.308)$ \\
\hline $\mathrm{J} 1935+1726$ & $90.7639\left( \pm 2 \times 10^{-5}\right)$ & $175.8( \pm 4.0)$ & $0.257(-0.107,+0.272)$ \\
\hline $\mathrm{J} 2229+2643$ & $93.0159\left( \pm 1.5 \times 10^{-7}\right)$ & $255.3( \pm 0.04)$ & $0.142(-0.057,+0.149)$ \\
\hline $\mathrm{J} 1751-2857$ & $110.7465\left( \pm 4 \times 10^{-8}\right)$ & $127.9( \pm 0.03)$ & $0.226(-0.093,+0.239)$ \\
\hline $\mathrm{J} 1853+1303$ & $115.6538\left( \pm 1.4 \times 10^{-8}\right)$ & $23.69( \pm 0.006)$ & $0.281(-0.119,+0.299)$ \\
\hline $\mathrm{J} 1955+2908$ & $117.3491\left( \pm 6 \times 10^{-8}\right)$ & $330.2( \pm 0.018)$ & $0.208(-0.085,+0.220)$ \\
\hline $\mathrm{J} 1529-3828$ & $119.6748\left( \pm 1.6 \times 10^{-5}\right)$ & $168.6( \pm 1.4)$ & $0.191(-0.078,+0.201)$ \\
\hline $\mathrm{J} 2302+4442$ & $125.9353\left( \pm 1.3 \times 10^{-7}\right)$ & $503.0( \pm 0.017)$ & $0.344(-0.148,+0.369)$ \\
\hline $\mathrm{J} 1643-1224$ & $147.0173\left( \pm 7 \times 10^{-5}\right)$ & $505.8( \pm 0.009)$ & $0.139(-0.055,+0.145)$ \\
\hline $\mathrm{J} 1708-3506$ & $149.1332\left( \pm 4 \times 10^{-7}\right)$ & $244.5( \pm 0.1)$ & $0.188(-0.076,+0.198)$ \\
\hline $\mathrm{J} 0214+5222$ & $512.0397\left( \pm 3 \times 10^{-4}\right)$ & $5.328 \times 10^{3}( \pm 0.5)$ & $0.483(-0.218,+0.528)$ \\
\hline $\mathrm{J} 0407+1607$ & $669.0704\left( \pm 1 \times 10^{-4}\right)$ & $936.8( \pm 0.6)$ & $0.223(-0.092,+0.235)$ \\
\hline $\mathrm{J} 2234+0611$ & $32.0014\left( \pm 1 \times 10^{-7}\right)$ & $1.293 \times 10^{5}( \pm 0.014)$ & $0.276( \pm 0.009)$ \\
\hline $\mathrm{J} 1946+3417$ & $27.0199\left( \pm 5 \times 10^{-8}\right)$ & $1.345 \times 10^{5}( \pm 0.017)$ & $0.2659( \pm 0.003)$ \\
\hline J1618-3921 & $22.7455\left( \pm 1.9 \times 10^{-7}\right)$ & $2.741 \times 10^{4}( \pm 1.0)$ & $0.2030(-0.083,+0.214)$ \\
\hline
\end{tabular}

Note.

${ }^{\text {a }}$ The errors of $M_{\mathrm{c}}$ for the pulsars marked with ${ }^{*}$ are adopted from van Kerkwijk et al. (2005) and references therein. For the others, assuming the pulsar mass to be $1.35 M_{\odot}$, their errors are estimated from the mass functions with the inclination angles varying from $i=90^{\circ}$ to $i=18^{\circ}$. 
Table 2

Orbital Properties of MSP Binaries with $\mathrm{CO} / \mathrm{ONeMg}$ WD Companions

\begin{tabular}{|c|c|c|c|}
\hline Pulsar Name ${ }^{a}$ & $\begin{array}{l}\text { Orbital Period } P_{\mathrm{b}} \\
\text { (days) }\end{array}$ & $\begin{array}{c}\text { Eccentricity } \epsilon \\
\left(10^{-6}\right)\end{array}$ & $\begin{array}{c}\text { Companion Mass } M_{\mathrm{c}} \\
\left(M_{\odot}\right)\end{array}$ \\
\hline $\mathrm{J} 1952+2630$ & $0.3919\left( \pm 7 \times 10^{-11}\right)$ & $40.85( \pm 0.1)$ & $1.133(-0.591,+1.379)$ \\
\hline $\mathrm{J} 1802-2124$ & $0.6989\left( \pm 5 \times 10^{-12}\right)$ & $2.474( \pm 0.5)$ & $0.78( \pm 0.04)$ \\
\hline $\mathrm{J} 1435-6100$ & $1.3549\left( \pm 1.8 \times 10^{-9}\right)$ & $10.47( \pm 1.5)$ & $1.079(-0.558,+1.301)$ \\
\hline $\mathrm{J} 1949+3106$ & $1.9495\left( \pm 2 \times 10^{-6}\right)$ & $43.06( \pm 0.07)$ & $0.967(-0.490,+1.143)$ \\
\hline J1439-5501 & $2.1179\left( \pm 3 \times 10^{-9}\right)$ & $49.85( \pm 1.5)$ & $1.376(-0.744,+1.749)$ \\
\hline $\mathrm{J} 1337-6423$ & $4.7853\left( \pm 5 \times 10^{-9}\right)$ & $19.85( \pm 0.09)$ & $0.949(-0.479,+1.119)$ \\
\hline $\mathrm{J} 1933+1726$ & $5.1539\left( \pm 2 \times 10^{-8}\right)$ & $67.45( \pm 10.0)$ & $0.942(-0.475,+1.109)$ \\
\hline J0721-2038 & $5.4608\left( \pm 8 \times 10^{-8}\right)$ & $102.0( \pm 5.0)$ & $0.548(-0.252,+0.605)$ \\
\hline $\mathrm{J} 1603-7202$ & $6.3086\left( \pm 5 \times 10^{-10}\right)$ & $9.338( \pm 0.005)$ & $0.338(-0.146,+0.362)$ \\
\hline $\mathrm{J} 1227-6208$ & $6.7210\left( \pm 4 \times 10^{-9}\right)$ & $1.149 \times 10^{3}( \pm 3.0)$ & $1.576(-0.875,+2.076)$ \\
\hline $\mathrm{J} 1022+1001$ & $7.8051\left( \pm 1.1 \times 10^{-6}\right)$ & $97.23( \pm 0.014)$ & $0.853(-0.422,+0.989)$ \\
\hline $\mathrm{J} 1933-6211$ & $12.8194\left( \pm 8 \times 10^{-10}\right)$ & $1.397( \pm 0.04)$ & $0.382(-0.167,+0.412)$ \\
\hline $\mathrm{J} 1750-2536$ & $17.1416\left( \pm 4 \times 10^{-6}\right)$ & $392.0( \pm 4.0)$ & $0.547(-0.252,+0.603)$ \\
\hline J0900-3144 & $18.7376\left( \pm 9 \times 10^{-10}\right)$ & $10.49( \pm 1.7)$ & $0.424(-0.188,+0.459)$ \\
\hline $\mathrm{J} 2045+3633$ & $32.2978\left( \pm 1 \times 10^{-6}\right)$ & $1.721 \times 10^{4}( \pm 0.05)$ & $0.955(-0.483,+1.127)$ \\
\hline $\mathrm{J} 1420-5625$ & $40.2945\left( \pm 4 \times 10^{-6}\right)$ & $3.500 \times 10^{3}( \pm 3.0)$ & $0.438(-0.195,+0.475)$ \\
\hline J1727-2946 & $40.3077\left( \pm 3 \times 10^{-8}\right)$ & $4.563 \times 10^{4}( \pm 0.16)$ & $1.007(-0.514,+1.199)$ \\
\hline $\mathrm{J} 1640+2224$ & $175.4607\left( \pm 7 \times 10^{-9}\right)$ & $797.3( \pm 0.013)$ & $0.290(-0.123,+0.309)$ \\
\hline
\end{tabular}

Note.

${ }^{\mathrm{a}}$ The errors of $M_{\mathrm{c}}$ for the pulsars marked with ${ }^{*}$ are adopted from van Kerkwijk et al. (2005) and references therein. For the others, assuming the pulsar mass to be $1.35 M_{\odot}$, their errors are estimated from the mass functions with the inclination angles varying from $i=90^{\circ}$ to $i=18^{\circ}$.

\section{ORCID iDs}

C. Y. Hui (i) https://orcid.org/0000-0003-1753-1660

A. K. H. Kong (i) https://orcid.org/0000-0002-5105-344X

\section{References}

Alpar, M. A., Cheng, A. F., Ruderman, M. A., \& Shaham, J. 1982, Natur, 300, 728 Antoniadis, J. 2014, ApJL, 797, L24

Antoniadis, J., Kaplan, D. L., Stovall, K., et al. 2016, ApJ, 830, 36

Archibald, A. M., Kaspi, V. M., Bogdanov, S., et al. 2010, ApJ, 722, 88

Archibald, A. M., Stairs, I. H., Ransom, S. M., et al. 2009, Sci, 324, 1411

Ashman, K., Bird, C., \& Zepf, S. 1994, AJ, 108, 2348

Backer, D. C., Kulkarni, S. R., Heiles, C., Davis, M. M., \& Goss, W. M. 1982, Natur, 300, 615

Backer, D. C., Kulkarni, S. R., \& Taylor, J. H. 1983, Natur, 301, 314

Barr, E. D., Freire, P. C. C., Kramer, M., et al. 2017, MNRAS, 465, 1711

Camilo, F. 1995, in The Lives of the Neutron Stars, ed. A. Alpar, U. Kizilouglu, \& J. van Paradis (Dordrecht: Kluwer), 243

Charbonnel, C., Däppen, W., Schaerer, D., et al. 1999, A\&AS, 135, 405

Dufour, P., Blouin, S., Coutu, S., et al. 2017, in ASP Conf. Ser. 509, 20th European White Dwarf Workshop, ed. P.-E. Tremblay, B. Gänsicke, \& T. Marsh (San Francisco, CA: ASP), 3

Eggleton, P. P. 1983, ApJ, 268, 368
Fabian, A. C., Pringle, J. E., Verbunt, F., \& Wade, R. A. 1983, Natur, 301, 222

Fraley, C., \& Raftery, A. E. 2002, J. Am. Stat. Assoc., 97, 611

Fraley, C., \& Raftery, A. E. 2007, J. Classification, 24, 155

Freire, P. C. C., \& Tauris, T. M. 2014, MNRAS, 438, L86

Hui, C. Y., Cheng, K. S., \& Taam, R. E. 2010, ApJ, 714, 1149

Maeder, A., \& Meynet, G. 1989, A\&A, 210, 155

Manchester, R. N., Hobbs, G. B., Teoh, A., \& Hobbs, M. 2005, AJ, 129, 1993

Nauenberg, M. 1972, ApJ, 175, 417

Octau, F., Cognard, I., Guillemot, L., et al. 2018, A\&A, 612, A78

Özel, F., \& Freire, P. C. C. 2016, ARA\&A, 54, 401

Phinney, E. S. 1992, RSPTA, 341, 39

Phinney, E. S., \& Kulkarni, S. R. 1994, ARA\&A, 32, 591

Radhakrishnan, V., \& Srinivasan, G. 1982, CSci, 51, 1096

Refsdal, S., \& Weigert, A. 1971, A\&A, 13, 367

Taam, R. E., King, A. R., \& Ritter, H. 2000, ApJ, 541, 329

Takata, J., Li, K. L., Leung, G. C. K., et al. 2014, ApJ, 785, 131

Tauris, T. M. 1996, A\&A, 315, 453

Tauris, T. M., \& Savonije, G. J. 1999, A\&A, 350, 928

Thorstensen, J. R., \& Armstrong, E. 2005, AJ, 130, 759

van Kerkwijk, M. H., Bassa, C. G., Jacoby, B. A., \& Jonker, P. G. 2005, in ASP Conf. Ser. 328, Binary Radio Pulsars, ed. F. A. Rasio \& I. H. Stairs (San Francisco, CA: ASP), 357

Wijnands, R., \& van der Klis, M. 1998, Natur, 394, 344 